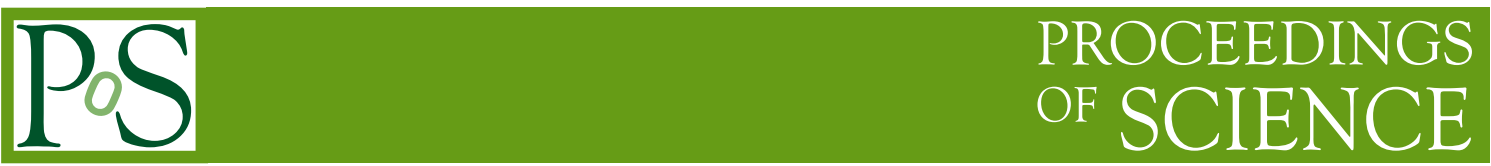

\title{
B meson excitations with chirally improved light quarks *
}

\author{
T. Burch $\dagger$, D. Chakrabarti, C. Hagen, T. Maurer, A. Schäfer \\ Institut für Theoretische Physik, Universität Regensburg, D-93040 Regensburg, Germany \\ E-mail: tommy.burch@physik.uni-regensburg.de \\ C. B. Lang and M. Limmer
}

Institut für Physik, FB Theoretische Physik, Universität Graz, A-8010 Graz, Austria

We present our latest results for the excitations of static-light mesons on both quenched and unquenched lattices, where the light quarks are simulated using the chirally improved (CI) lattice Dirac operator.

The XXV International Symposium on Lattice Field Theory 30 July - 4 August 2007

Regensburg, Germany

${ }^{*}$ For the BGR Collaboration.

† Speaker. 


\section{Light-quark propagator estimation}

To enhance the signals of our static-light correlators, we use an improved estimate of the lightquark propagator from any point within half of the lattice to any point in the other half. This so-called "domain decomposition improvement" was outlined and tested in Ref. [1] and amounts to a variant of the "maximal variance reduction" approach [2]. We present the basics of the method here.

Decomposing the lattice into two distinct domains, the full Dirac matrix can be written in terms of submatrices

$$
M=\left(\begin{array}{ll}
M_{11} & M_{12} \\
M_{21} & M_{22}
\end{array}\right),
$$

where $M_{11}$ and $M_{22}$ connect sites within a region and $M_{12}$ and $M_{21}$ connect sites from the different regions. We can also write the propagator in this form:

$$
M^{-1}=P=\left(\begin{array}{ll}
P_{11} & P_{12} \\
P_{21} & P_{22}
\end{array}\right) .
$$

The propagator between regions 1 and 2 is then estimated using $N$ random sources $\left(\chi^{n}, n=1, . ., N\right)$ :

$$
\begin{aligned}
P_{12} & =-M_{11}^{-1} M_{12} P_{22} \\
& \approx-M_{11}^{-1} M_{12} \frac{1}{N} \chi_{2}^{n} \chi_{2}^{n \dagger} P \\
& \approx-\frac{1}{N}\left(M_{11}^{-1} M_{12} \chi_{2}^{n}\right)\left(\gamma_{5} P \gamma_{5} \chi_{2}^{n}\right)^{\dagger} \equiv-\frac{1}{N} \psi_{1}^{n} \phi_{2}^{n \dagger} .
\end{aligned}
$$

Note that no sources are needed in region 1 and those in region 2 should reach region 1 with one application of $M$. Since $M$ is usually a sparse matrix, this greatly reduces the number of lattice sites which the random sources cover. ${ }^{1}$

In the following, we use the chirally improved (CI) lattice Dirac operator [3] for $M$.

\section{Static-light correlators}

Using different "wavefunctions" for the light-quark source and sink, we construct the following matrix of correlators:

$$
\begin{aligned}
C_{i j}(t) & =\left\langle 0\left|\left(\bar{Q} O_{j} q\right)_{t}\left(\bar{q} \bar{O}_{i} Q\right)_{0}\right| 0\right\rangle \\
& =\left\langle\sum_{x} \operatorname{Tr}\left[\frac{1+\gamma_{4}}{2} \prod_{k=0}^{t-1} U_{4}^{\dagger}(x+k \hat{4}) O_{j} P_{x+t \hat{4}, x} \bar{O}_{i}\right]\right\rangle,
\end{aligned}
$$

where $x$ is in one domain and $x+t \hat{4}$ is in the other.

We use bilinears of the form:

$$
\bar{Q} O_{j} q=\bar{Q} O(\Gamma, \vec{D})\left(\vec{D}^{2}\right)^{l_{j}} S_{J}\left(\kappa, N_{s m, j}\right) q,
$$

\footnotetext{
${ }^{1}$ Even for the case of a non-sparse lattice Dirac operator (Overlap or low-mode-subtracted), one may separate (or "dilute") the sources into, for example, those close to the boundary and those further away: e.g., $\chi_{2}^{n}=\chi_{2, t=t_{\text {bound }}}^{n}+$ $\chi_{2, t>t_{\text {bound }}}^{n}$.
} 


\begin{tabular}{lcc}
\hline oper. & $J^{P}$ & $O(\Gamma, \vec{D})$ \\
\hline$S$ & $0^{-}, 1^{-}$ & $\gamma_{5}$ \\
$P_{-}$ & $0^{+}, 1^{+}$ & $\sum_{i} \gamma_{i} D_{i}$ \\
$P_{+}$ & $1^{+}, 2^{+}$ & $\gamma_{1} D_{1}-\gamma_{2} D_{2}$ \\
$D_{ \pm}$ & $1^{-}, 2^{-}, 3^{-}$ & $\gamma_{5}\left(D_{1}^{2}-D_{2}^{2}\right)$ \\
\hline
\end{tabular}

Table 1: Static-light meson operators.

\begin{tabular}{|c|c|c|c|c|c|}
\hline$N_{S}^{3} \times N_{T}$ & $a(\mathrm{fm})$ & $M_{\pi, \text { sea }}$ & link smear & $N_{\text {conf }}$ & $\left(\begin{array}{llll}l_{1} & l_{2} & N_{s m, 1} & N_{s m, 2} \\
l_{3} & l_{4}, & N_{s m, 3} & N_{s m, 4}\end{array}, \kappa\right)$ \\
\hline $12^{3} \times 24$ & 0.20 & $\infty$ & Hyp & 200 & $\left(\begin{array}{lllll}0 & 0 & 0 & 8 \\
1 & 2 & 12 & 16 & 0.2\end{array}\right)$ \\
\hline $16^{3} \times 32$ & 0.15 & $\infty$ & Нyр & 100 & $\left(\begin{array}{llll}0 & 0 & 0 & 12 \\
1 & 2 & 18 & 24\end{array}, 0.2\right)$ \\
\hline $16^{3} \times 32$ & 0.16 & $450 \mathrm{MeV}$ & Stout & 40 & $\left(\begin{array}{llll}0 & 0 & 0 & 12 \\
1 & 2\end{array}, \begin{array}{ll}18 & 24\end{array}, 0.2\right)$ \\
\hline
\end{tabular}

Table 2: Parameters for the configurations and quark source smearings.

where $S_{J}$ is a gauge-covariant (Jacobi) smearing function and we apply $l_{j}=0,1$, or 2 Laplacians. We also include the local source to obtain a $4 \times 4$ correlator matrix for each set of quantum numbers, determined by $O(\Gamma, \vec{D})$ (see Table $\mathbb{1}$ ). The parameters used for smearing the light-quark sources and the details of the configurations we use [4] are given in Table 2 .

\section{Mass splittings}

Once we have our correlator matrices, we apply the variational method [5] and solve the generalized eigenvalue problem

$$
\sum_{j} C_{i j}(t) v_{j}^{k}=\lambda^{k}\left(t, t_{0}\right) \sum_{j} C_{i j}\left(t_{0}\right) v_{j}^{k}
$$

The eigenvalues behave as

$$
\lambda^{k}\left(t, t_{0}\right) \propto e^{-t M_{k}}\left[1+O\left(e^{-t \Delta M_{k}}\right)\right],
$$

where $\Delta M_{k}$ is the difference to the state closest in mass to $M_{k}$. To help stabilize the matrix diagonalization, we check that our correlator matrices are real and symmetric (within errors) and then symmetrize them before solving the eigenvalue problem (via Cholesky decomposition). Although in principle one should work at the largest possible value of $t_{0}$, we find a negligible $t_{0}$-dependence in the eigenvalues and effective masses (and their jackknife errors) over the region where it is still possible to invert $C\left(t_{0}\right)$. So we present results where $t_{0} / a=1$.

In Fig. 1 we show some of the effective masses which result from the $16^{3} \times 32$ quenched configurations. Figure 1 displays the effective masses from the dynamical configurations. In each figure appear the $S-, P_{--}, P_{+^{-}}$, and $D_{ \pm}$-waves from left to right, respectively. The horizontal lines represent $M \pm \sigma_{M}$ fit values for the corresponding time ranges.

Figure 3 displays the mass differences $M-M_{1 S}$ on the $16^{3} \times 32$ quenched lattice as a function of the light-quark mass. The green crosses at $m_{q}=m_{s}$ and $m_{q}=0$ are the experimental masses 

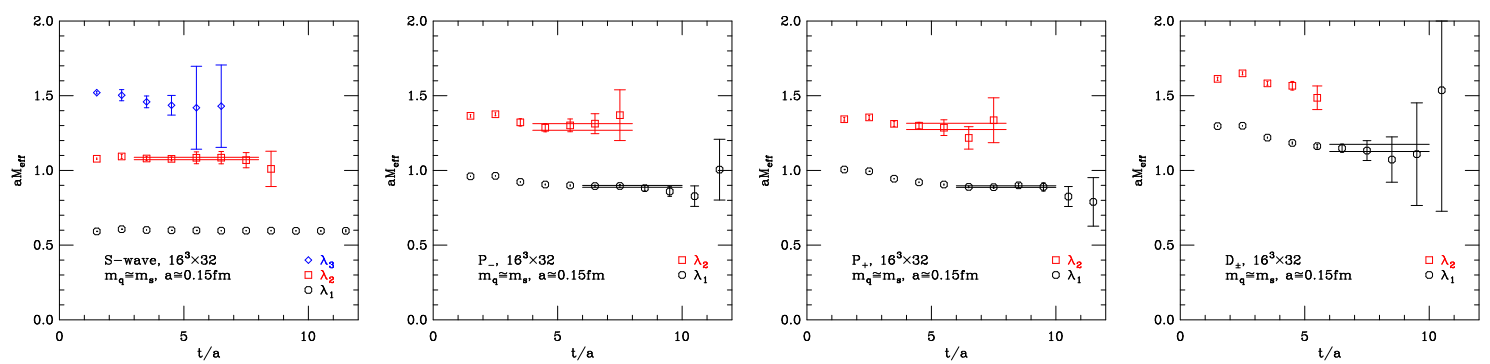

Figure 1: Effective masses for the static-light mesons on the quenched configurations. $a m_{q}=0.08, a^{-1} \approx$ $1330 \mathrm{MeV}, L \approx 2.4 \mathrm{fm}$. The horizontal lines represent $M \pm \sigma_{M}$ fit values for the corresponding time ranges.
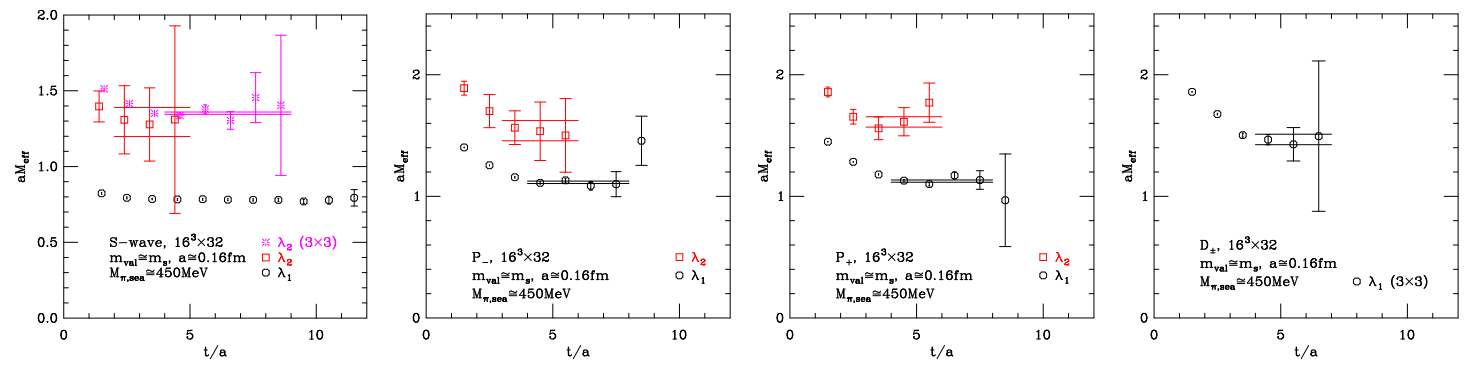

Figure 2: Effective masses for the static-light mesons on the dynamical configurations. $m_{q} \approx m_{s}, a^{-1} \approx 1230$ $\mathrm{MeV}, M_{\pi, \text { sea }} \approx 450 \mathrm{MeV}, L \approx 2.5 \mathrm{fm}$. The horizontal lines represent $M \pm \sigma_{M}$ fit values for the corresponding time ranges.

for $B_{s J}^{*}$ and $B_{J}^{*}$ (differenced from $3 M_{B_{(s)}^{*}} / 4+M_{B_{(s)}} / 4$ ), respectively, taken from the PDG [6]. The black symbols at $m_{q}=m_{s}$ are the results from the dynamical lattice. The symbols have the same meaning as the colored ones for the quenched case.

\section{Discussion}

In Table 3 we report our $B_{s}$ meson mass splittings in physical units for the three lattices considered. One can see here that the $1 P-1 S$ splitting is too small when compared with experiment (as opposed to the $m_{q} \rightarrow 0$ case, where it appears too high; see Fig. 3). Also, with statistical errors of $\sim 10 \mathrm{MeV}$, the $1 P_{+}-1 P_{-}$splitting is not resolved, except on the coarser quenched lattice, where it is $\sim 40 \mathrm{MeV}$. We plan to study this further with a finer quenched lattice and higher statistics for the dynamical lattice. We would also like to try to include $1 / m_{Q}$ effects by interpolating between our results $\left(m_{Q}=\infty\right)$ and the experimental results for $D_{s}$ mesons (see [7]).

It will also be interesting to watch the $2 S-1 S$ splitting (holding thus far $\sim 650-700 \mathrm{MeV}$ for $m_{q} \approx m_{s}$; see also [1]) as we proceed to higher statistics and finer lattice spacing.

It is important to keep in mind the possibly additional systematic error introduced by setting the scale of our lattices (we use $\left.r_{0}=0.5 \mathrm{fm}\right)$. A smaller value $\left(r_{0} \approx 0.45-0.5 \mathrm{fm}\right.$; see, e.g., [8]) would enhance our mass splittings. 


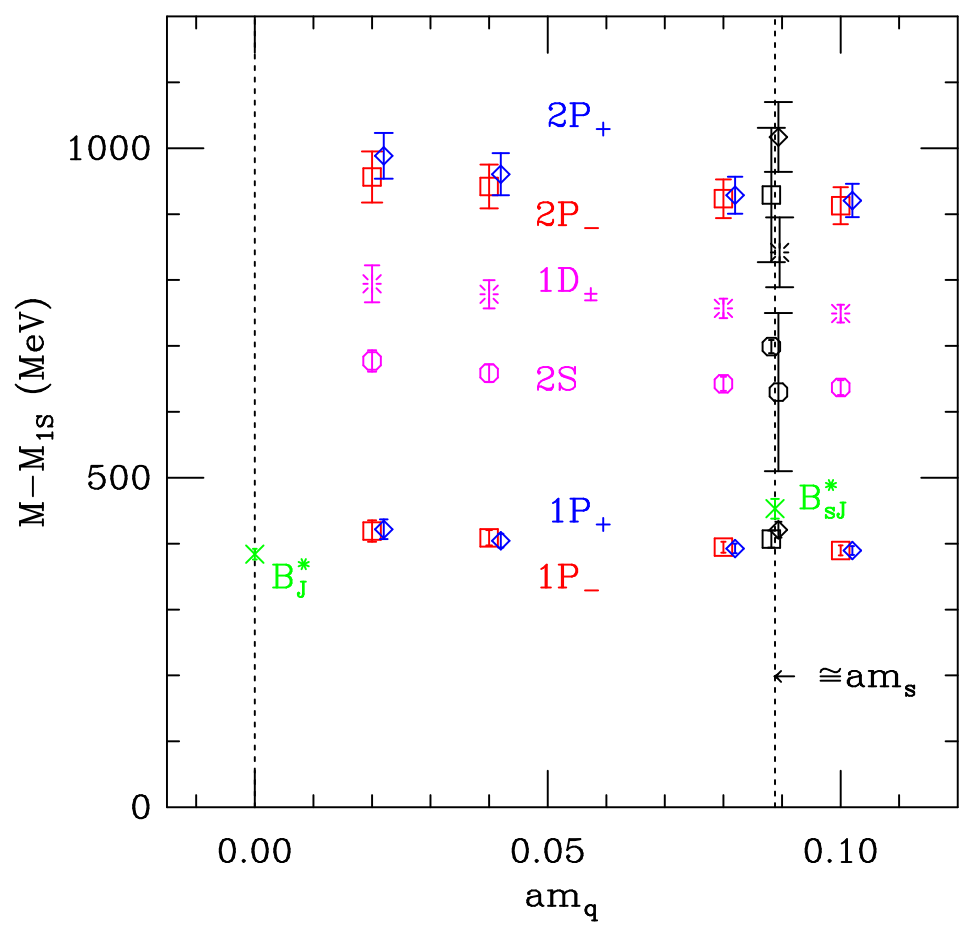

Figure 3: Mass splittings $\left(M-M_{1 S}\right)$ in $\mathrm{MeV}$ as a function of the quark mass for the $16^{3} \times 32$ lattices. The black points at $m_{s}$ represent the results from the dynamical lattice. The squares are for $P_{-}$results, diamonds are for $P_{+}$, circles are for $2 S$, and bursts are for $1 D_{ \pm}$. The green $\times$'s are the experimental masses for $B_{(s) J}^{*}$ (differenced from $3 M_{B_{(s)}^{*}} / 4+M_{B_{(s)}} / 4$ ) from the PDG [6].

\begin{tabular}{lcccc}
\hline state & \multicolumn{4}{c}{$M-M_{1 S}(\mathrm{MeV})$} \\
& $M_{\pi, \mathrm{sea}}=\infty$ & $M_{\pi, \mathrm{sea}}=\infty$ & $M_{\pi, \mathrm{sea}} \approx 450 \mathrm{MeV}$ & PDG [6] \\
& $a \approx 0.20 \mathrm{fm}$ & $a \approx 0.15 \mathrm{fm}$ & $a \approx 0.16 \mathrm{fm}$ & \\
\hline $2 S$ & $684(14)$ & $640(11)$ & $699(10)\left(_{-180}^{+40}\right)$ & - \\
\hline $1 P_{-}$ & $385(7)$ & $393(8)$ & $407(13)$ & $453(15)$ \\
$2 P_{-}$ & $995(20)$ & $918(29)$ & $930(100)$ & - \\
\hline $1 P_{+}$ & $422(4)\left(_{-7}^{+0}\right)$ & $391(7)$ & $421(12)$ & $453(15)$ \\
$2 P_{+}$ & $967(17)$ & $925(27)$ & $1017(53)$ & - \\
\hline $1 D_{ \pm}$ & $730(12)$ & $755(14)$ & $842(53)$ & - \\
$2 D_{ \pm}$ & $1210(30)$ & - & - & - \\
\hline
\end{tabular}

Table 3: Our static-light meson mass splittings at $m_{q}=m_{s}$. Numbers in the first set of parentheses are statistical errors. The second set (if present) represent the changes in the error bounds when shifting to another seemingly good fit range (for the dynamical $2 S$ state, this is accompanied by an increase in the basis from the first 3 operators to the full 4). 


\section{Acknowledgments}

We would like to thank Christof Gattringer for helpful discussions. Simulations were performed at the LRZ in Munich. This work is supported by GSI. The work of D.C. is supported by the Alexander von Humboldt Foundation.

\section{References}

[1] T. Burch and C. Hagen, Comput. Phys. Commun. 176, 137 (2007).

[2] C. Michael and J. Peisa, Phys. Rev. D 58 (1998) 034506.

[3] P. H. Ginsparg and K. G. Wilson, Phys. Rev. D 25 (1982) 2649; C. Gattringer, Phys. Rev. D 63 (2001) 114501 [hep-lat/0003005]; C. Gattringer, I. Hip, and C. B. Lang, Nucl. Phys. B 597 (2001) 451 [hep-lat/0007042].

[4] C. B. Lang, P. Majumdar, and W. Ortner, Phys. Rev. D 73, 034507 (2006); C. B. Lang, et al., PoS (LATTICE 2007) 114.

[5] C. Michael, Nucl. Phys. B 259 (1985) 58; M. Lüscher and U. Wolff, Nucl. Phys. B 339 (1990) 222; T. Burch, C. Gattringer, L. Y. Glozman, C. Hagen, and C. B. Lang, Phys. Rev. D 73, 017502 (2006).

[6] W.-M. Yao, et al., Journal of Physics G 33, 1 (2006).

[7] A. M. Green, J. Koponen, C. McNeile, C. Michael, and G. Thompson, Phys. Rev. D 69, 094505 (2004).

[8] C. McNeile, PoS (LATTICE 2007) 019. 